predicted probabilities (Hosmer-Lemeshow test, $\mathrm{p}=0.818$ ). In the high-risk group ( $\mathrm{n}=34)$, the predicted probability of LN metastasis was $53.0 \%$ and the actual rate of $\mathrm{LN}$ metastasis was $50.0 \%$. In the low-risk group, 11 of 16 patients with LN metastasis had the tumor location in the cornu $(n=7)$ and lower uterine segment $(n=4)$.

Conclusion: Approximately $90 \%$ of presumed early-stage endometrioid EC patients may not require lymphadenectomy and this nomogram may provide valuable guidance for physicians on the lymphadenectomy. In the low-risk group, the tumor location including cornu and lower uterine segment should be investigated to determine whether to perform lymphadenectomy.

Poster (E27)

Endometrial Hyperplasia, Endometrial Intra-epithelial Neoplasia, and Endometrial Cancer

https://doi.org/10.3802/jgo.2021.32.S1.E27

\section{Outcome of therapeutic lymph node dissection for stage IIIC2 uterine cancer}

Tomoyasu Kato, 'Syoichi Kitamura, Risako Ozawa, Takashi Natsume, Erisa Fujii, Mayumi Kato, Yasuhito Tanase, Masaya Uno, Mitsuya Ishikawa

Cancer Center Hospital, Tokyo, Japan (tokato@ncc.go.jp)

Objective: The purpose of this study was to compare the treatment outcomes of stage IIIC2 uterine cancer in our department by histological type.

Methods: Thirty-three cases of stage IIIC2 uterine cancer in which lymph node dissection (LND) was performed at our department from 2006 to 2017 were included in this study. LND was conducted with sharp dissection between the vascular sheath and the adventitia of the vessels. Paraaortic lymph node (PAN) biopsy cases were excluded. Postoperative adjuvant chemotherapy was administered. The histological types were divided into estrogen-dependent Type I (endometrioid, grade 1 to 3), estrogen-independent type II, and carcinosarcoma, and the overall survival times were compared.

Results: The median (range) number of PAN metastases was 2 (1-15), 4 (1-19), and 4 (2-34), respectively. The median number of pelvic lymph node metastases was 2, 4, and 3, respectively. There were no cases of postoperative chylous ascites. Three cases of type I, 3 cases of type II, and all cases of carcinosarcoma recurred. The 5 -year survival was $76 \%$ overall, $89 \%$ for type I, $78 \%$ for type II, and 33\% for carcinosarcoma. There was no significant difference among the 3 groups, but there was a significant difference between the 2 groups of type I and type II
+ carcinosarcoma group.

Conclusion: With our systemic LND, the 5-year survival rate for stage IIIC2 type 1 patients with a median of 2 positive PANs was as high as $89 \%$. Our sharp LND is safe and has high therapeutic value.

Poster (E28)

Gestational Trophoblastic Neoplasia

https://doi.org/10.3802/jgo.2021.32.S1.E28

\section{Persistent low concentration hCG in gestational trophoblastic neoplasia with history of 7 series methotrexate}

\author{
Veinardi Madjid, ${ }^{1}$ Kartiwa Hadi Nuryanto2,* \\ 'Dharmais Cancer Hospital, Jakarta, Indonesia \\ ${ }^{2}$ Dr. Cipto Mangunkusumo National Central Referral Hospital, Jakarta, \\ Indonesia (kartiwa_h_nuryanto@gmail.com)
}

Objective: Persistently low concentration of human chorionic gonadotropin (hCG) may be associated with false-positive hCG, quiescent gestational trophoblastic disease and pituitary hCG. The knowledge about it, is needed to give the best option to the patient.

Methods: This is a case report of patient Mrs. GM, 24-yearold, with history of referred from Secondary Public Hospital with gestational trophoblastic neoplasia (GTN). The patient had a history of curettage due to molar pregnancy in February 2018, without any hCG level data stated before curettage. We evaluated the hCG measurement after curettage, during single agent chemotherapy methotrexate (MTX) and after unresponsiveness single agent chemotherapy MTX.

Results: Mrs. GM's hCG level before single-agent chemotherapy was $260.7 \mathrm{IU} / \mathrm{mL}$. She underwent single-agent chemotherapy MTX for 7 consecutive series. The hCG levels ( $\mathrm{mIU} / \mathrm{mL}$ ) during single-agent were 37.2, 14.7, 6.3, 6.4, 5.7, 4.6 and 5.1. The hCG level after the 7th MTX increased to $5.1 \mathrm{mIU} / \mathrm{mL}$. Therefore, the patient was sent to Dr. Cipto Mangunkusumo National Central Referral Hospital (RSCM) due to unresponsiveness to single-agent chemotherapy on GTN. No complaint, neither any remarkable findings on physical examination in RSCM. Internal genitalia was normal in abdominal and trans-vaginal ultrasound. We also found normal chest X-ray examination. The patient was diagnosed with false-positive hCG and planned for continuing hCG observation. After 2 to 4 months of observation, the hCG level decreased to $4 \mathrm{mIU} / \mathrm{mL}, 4.3 \mathrm{mIU} / \mathrm{mL}$ and $2.46 \mathrm{mIU} / \mathrm{mL}$.

Conclusion: The hCG levels returned to normal and further chemotherapy was no longer needed. 